occur in any platyrrhine", which is contrary to fact ; and to give another example, he talks of "lethal factors" in a genetic content, in a way which suggests that he does not realize how much biometrical study needs to be undertaken before such things can be posited.

But having said all this, the fact remains that, in spite of these faults, the book contains a mass of good meat. Its very presence and massiveness will ensure it, as the same qualities will their predecessors, a useful life.

\section{ELECTRICAL SCIENCE}

Introduction to Electrical Applied Physics

By Prof. N. F. Astbury. Pp. xi +241. (London : Chapman and Hall, Ltd., 1956.) 36s. net.

A

$\mathrm{S}$ the author says in the preface to his book, it is an attempt to bring together the classical bases of electrical science and the elements of some specialist topics which, by the natural processes of technological evolution, are finding their way into all fields of applied science.

The method of presentation used throughout the book is to deal first with the fundamental physical and theoretical principles of each topic and then to apply them to the consideration of practical problems. Special mathematical tools such as space vectors, Bessel functions and Heaviside's operational analysis are treated with their applications, rather than abstractly and in a separate part of the book. This feature will be particularly welcomed by the reader with a practical rather than an analytical turn of mind. Consequently, each chapter is almost selfcontained and the necessity for cross-referencing is much reduced. But where it is still needed, the task might have been simplified by the simple expedient of marking the chapter and section numbers on each page.

The book begins with a consideration of the physical basis of the subject, introducing the concepts of the quantum theory when dealing with electric conduction in solids, treating the origin of magnetism from the point of view of the domain theory, and briefly outlining the behaviour of dielectrics in terms of polar and non-polar molecules. Electric and magnetic fields are treated on classical lines. In a chapter on electrical units and the general field equations, a sound case is made for using a rational system of units which gives the general equations a homogeneous form; the rationalized M.K.S. system is used throughout the book. An examination of the field equations when conduction current is present leads to expressions for the penetration of flux, and for the eddy-current loss, in flat sheets and circular cylinders, and for the skin effect in cylindrical conductors; Bessel functions are introduced to the reader unfamiliar with them as the solutions to familiar problems.

The chapter on electric circuits deals with lines, filters and general methods of circuit analysis including the use of the Laplace transform. (The use of the lower case symbol $i$ for the electric current vector in equations such as $V / i=R+j X$ should be altered in a reprint of the book.)

Gapped magnetic circuits, including permanent magnets, the equivalent circuit of an iron-cored coil system, distortion and the magnetic amplifier, are treated in an excellent chapter on magnetic circuits. As in other parts of the book, the author has succeeded in presenting the basic physical facts and principles so that they seem to lead naturally to important practical applications.

In connexion with electro-mechanical and electroacoustical systems, limitations of sensitivity due to noise are considered, and a brief introduction is given to the study of servo-mechanisms. The chapter on electron devices deals with the deflexion of electron beams, thermionic valves and valve amplifiers, transistors and photosensitive devices. The final chapter deals with electro-magnetic waves and radiation, including a study of transmission through the ionosphere and an introduction to the theory of wave guides.

As the author modestly observes in the preface, "the subject of every chapter in this book forms, elsewhere, the subject of a separate treatise", and those reconmended by the author are listed in the book. Unfortunately, no other references are given.

The work is intended for the senior university student in physics or engineering, but it should also appeal to others seeking an understanding of the principles underlying many recent technological developments. It is easy to read, well produced and reasonably priced.

H. Kayser

\section{NUMERICAL METHODS}

Numerical Methods

By Dr. R. A. Buckingham. Pp. xii +597. (London : Sir Isaac Pitman and Sons, Ltd., 1957.) 70s. net.

$\mathrm{N}$ recent years a number of books on numerical analysis have been brought before the scientific public, and these vary in their objectives from educational didactics to automatic digital calculators, and in their style from lucidity to the pomposity which besets certain authors when they come to expound their so-called 'philosophy'. Dr. R. A. Bucking. ham's book is the latest work on the subject to come before the reviewer and, although the publishers' lists seem to give promise of more such works in the future, it will be difficult for authors to find new ground to cover, at least on the general plane.

To the last statement certain limited qualifications are necessary. Two omissions from his book are mentioned by Dr. R. A. Buckingham in his preface : namely, discussion of Gaussian methods of quadrature and of the Runge-Kutta formulæ for the solution of differential equations. It is a little difficult to accept the author's excuse that these omissions are justified by the presence of an adequate literature in the field, because this applies to much that he does include. A further omission, which is rather more difficult to understand, is that of an up-to-date discussion of throwback, particularly in view of the author's plea that the book is intended for hand computers. On a minor plane, I regret the absence of an account of vector-space methods for the solution of simultaneous equations, linear and otherwise.

These criticisms are, however, ones of detail and should not detract from the overall good impression which the contents of the book produce. The treat. ment is to some extent classical. Thus, the first five chapters discuss errors, tables and desk machines; 